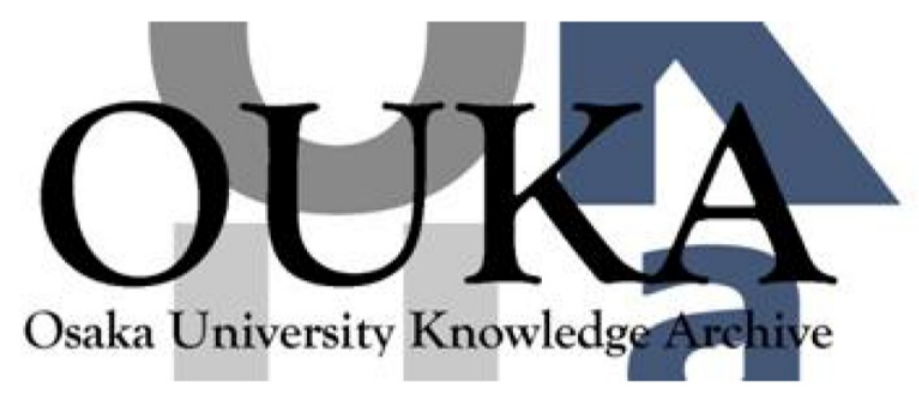

\begin{tabular}{|c|l|}
\hline Title & $\begin{array}{l}\text { Modulated photoconductivity study of charged } \\
\text { and neutral defects in undoped amorphous } \\
\text { silicon }\end{array}$ \\
\hline Author(s) & Hattori, K.; Adachi, Y.; Anzai, M. et al. \\
\hline Citation & Journal of Applied Physics. 76(5) p. 2841-p. 2850 \\
\hline Issue Date & $1994-09-01$ \\
\hline oaire:version & VoR \\
\hline URL & https://hdl. handle. net/11094/2993 \\
\hline rights & \\
\hline Note & \\
\hline
\end{tabular}

Osaka University Knowledge Archive : OUKA

https://ir. Library. osaka-u. ac. jp/

Osaka University 


\title{
Modulated photoconductivity study of charged and neutral defects in undoped amorphous silicon
}

\author{
K. Hattori, Y. Adachi, M. Anzai, H. Okamoto, and Y. Hamakawa \\ Faculty of Engineering Science, Osaka University, Toyonaka, Osaka 560, Japan
}

(Received 4 January 1994; accepted for publication 23 May 1994)

\begin{abstract}
A novel spectroscopy tcchnique based on modulated photoconductivity measurements with varying illumination level has been applied to investigate the capture coefficients and the energy distribution of defect states in undoped amorphous silicon. From the experimental data, charged and neutral defect distributions are clearly resolved according to their own capture coefficients. The carrier capture process as well as the defect formation mechanism are both quantitatively discussed.
\end{abstract}

\section{INTRODUCTION}

Defect states in hydrogenated amorphous silicon ( $a$ $\mathrm{Si}: \mathrm{H})$ act as trapping as well as recombination centers and affect the performance of the electronic devices, such as solar cells ${ }^{1}$ and thin-film transistors, ${ }^{2}$ based on this material. A variety of methods have been utilized to measure the density of defect states in $a-\mathrm{Si}: \mathrm{H}^{3}$ Among them, a junction capacitance transient $(\mathrm{JCT})^{4}$ yields the most straightforward and reliable assessments. The JCT measurements, which are normally applied to the doped samples, monitor charge transients due to carrier thermal emissions from the defect states which are initially populated with carriers by a voltage filling pulse, profiling the occupied defect distribution. On the other hand, the technique based on measurements of modulated photoconductivity (MPC) ${ }^{5-12}$ that reflects the trapping and release kinetics of photocarriers has attracted popularity recently. Application of the MPC method can determine the distributions of defects unoccupied under the dark equilibrium versus a thermal emission energy scale. Thus both techniques are thought to be complementary to each other.

In $a-\mathrm{Si}: \mathrm{H}$, there is now considerable experimental evidence that dominant defect states originate from $\mathrm{Si}$ dangling bonds, and that the distribution of these states is determined, following a thermal equilibrium description that involves a broad band of energy levels available for the formation of a defect, the so-called defect pool. ${ }^{2,13-16}$ The defect pool concept leads to the defect bands being shifted energetically from each other, depending on the charge state. The intensity of these defect bands is essentially correlated to the Fermilevel location, resulting in the density of states being dominated by negatively charged dangling bonds $\left(D^{-}\right)$in $n$-type $a$-Si:H and by the positively charged dangling bonds $\left(D^{+}\right)$in $p$-type $a$-Si:H. The single $D^{-}$distribution has been identified by JCT spectroscopy measurements. ${ }^{4}$

As for intrinsic $a-\mathrm{Si}: \mathrm{H}$, a concurrence of the charged $D^{-,+}$and neutral $D^{0}$ dangling bonds is inferred theoretically. ${ }^{15,16}$ An experimental identification for the composite distribution of $D^{-, 0,+}$ states, however, remains far from being achieved. A prime candidate for approaching the problem inherent in intrinsic materials is an application of the MPC spectroscopy. Clearly required is not only a resolution with respect to the thermal cmission encrgy of the defect but also one with respect to the capture efficiencies expected to be different between the charged and neutral defects. In this article, we develop the MPC theory for the case in which different defects species are involved, propose an experimental technique for measuring their capture efficiencies and describe the results of measurements on undoped $a-\mathrm{Si}: \mathrm{H}$ as well as some physical insights obtained from it.

\section{THEORY OF MODULATED PHOTOCONDUCTIVITY (MPC) SPECTROSCOPY}

\section{A. General analysis of MPC}

Considered here is an amorphous semiconductor thin film illuminated by a weakly absorbed band-gap light so that concentrations of electrons $(n)$ and holes $(p)$ in the extended states are uniform in space. The continuity equations for $n$ and $p$ should be formulated by taking into account electronic transitions that can occur between the extended states and the localized states distributed within the band gap. ${ }^{17}$ Let us suppose that the localized states arise from the defect species labeled by $j$, each with characteristic coefficients $C_{n, p}^{j}$ describing the capture efficiencies for electrons and holes, respectively, then the corresponding expressions are written as

$$
\frac{d n}{d t}+\sum_{j} \int_{\epsilon_{v}}^{\epsilon_{c}} d \epsilon D^{j}(\epsilon)\left\{n C_{n}^{j}\left[1-f^{j}(\epsilon)\right]-e_{n}^{j}(\epsilon) f^{j}(\epsilon)\right\}=G
$$

$\frac{d p}{d t}+\sum_{j} \int_{\epsilon_{v}}^{\epsilon_{c}} d \epsilon D^{j}(\epsilon)\left\{p C_{p}^{j} f^{i}(\epsilon)-e_{p}^{j}(\epsilon)\left[1-f^{j}(\epsilon)\right]\right\}=G$

Here, $t$ is the time, $G$ is the photogeneration rate of electronhole pairs, $D^{j}(\epsilon)$ is the density of defects states at the energy $\epsilon$, and $e_{n, p}^{j}(\epsilon)$ are the thermal emission rates for electrons and holes, respectively, given by

$$
\begin{aligned}
& e_{n}^{j}(\epsilon)=N_{c} C_{n}^{j} \exp \left(-\frac{\epsilon_{c}-\epsilon}{k T}\right), \\
& e_{p}^{j}(\epsilon)=N_{v} C_{p}^{j} \exp \left(-\frac{\epsilon-\epsilon_{v}}{k T}\right),
\end{aligned}
$$

where $k T$ is the thermal energy, $N_{c, v}$ the effective densities of conduction band states above $\epsilon_{c}$ and of valence band states below $\epsilon_{v}$, respectively. The occupation function $f^{j}(\epsilon)$ obeys the rate equation 
$\frac{d f^{j}(\epsilon)}{d t}=\left[n C_{n}^{j}+e_{p}^{j}(\epsilon)\right]\left[1-f^{j}(\epsilon)\right]-\left[p C_{p}^{j}+e_{n}^{j}(\epsilon)\right] f^{j}(\epsilon)$.

In the MPC experiment, the excitation light sinusoidally varying in timc is used such that

$$
G=G_{0}+G_{\omega} \cos (\omega t) .
$$

Solution forms for carrier concentrations that assume a linear response to generation

$$
\begin{aligned}
& n=n_{0}+\operatorname{Re}\left[n_{\omega} \exp (i \omega t)\right], \\
& p=p_{0}+\operatorname{Re}\left[p_{\omega} \exp (i \omega t)\right]
\end{aligned}
$$

are successfully applied in a small signal case $\left(n_{\omega} \ll n_{0}\right.$ and $\left.p_{\omega} \& p_{0}\right),{ }^{9}$ where $i^{2}=-1, n_{0}$ and $p_{0}$ are the steady-state concentrations generated by $G_{0}$, and $n_{\omega}$ and $p_{\omega}$ are complex numbers that give the amplitudes and phases of sinusoidal parts of concentrations. The resulting photoconductivity is represented by

$$
\sigma=\sigma_{0}+\operatorname{Re}\left[\sigma_{\omega} \exp (i \omega t)\right],
$$

where $\sigma_{0, \omega}=e\left(\mu_{n} n_{0, \omega}+\mu_{p} p_{0, \omega}\right), e$ is the unit electronic charge, and $\mu_{n, p}$ the mobilities of electrons and holes, respectively. Substituting Eqs. (4)-(6) into Eqs. (1)-(3) yields

$\frac{n_{\omega}}{G_{\omega}}=\frac{i \omega+P}{(i \omega+N)(i \omega+P)+(i \omega+N) \bar{P}+(i \omega+P) \bar{N}}$,

$\frac{p_{\omega}}{G_{\omega}}=\frac{i \omega+N}{(i \omega+N)(i \omega+P)+(i \omega+N) \bar{P}+(i \omega+P) \bar{N}}$.

Parameters involved in the right-hand side of the equations are defined by the following:

$$
\begin{aligned}
& N=\sum_{j} \int_{\epsilon_{v}}^{\epsilon_{c}} d \epsilon C_{n}^{j} D^{j}(\epsilon)\left[1-f_{0}^{j}(\epsilon)\right] \frac{i \omega}{i \omega+R_{0}^{j}(\epsilon)}, \\
& P=\sum_{j} \int_{\epsilon_{v}}^{\epsilon_{c}} d \epsilon C_{p}^{j} D^{j}(\epsilon) f_{0}^{j}(\epsilon) \frac{i \omega}{i \omega+R_{0}^{j}(\epsilon)}, \\
& \bar{N}=\sum_{j} \int_{\epsilon_{v}}^{\epsilon_{\epsilon}} d \epsilon C_{n}^{j} D^{j}(\epsilon)\left[1-f_{0}^{j}(\epsilon)\right] \frac{p_{0} C_{p}^{j}+e_{p}^{j}(\epsilon)}{i \omega+R_{0}^{j}(\epsilon)}, \\
& \bar{P}=\sum_{j} \int_{\epsilon_{v}}^{\epsilon_{c}} d \epsilon C_{p}^{j} D^{j}(\epsilon) f_{0}^{j}(\epsilon) \frac{n_{0} C_{n}^{j}+e_{n}^{j}(\epsilon)}{i \omega+R_{0}^{j}(\epsilon)} .
\end{aligned}
$$

The steady-state occupation function $f_{0}^{j}(\epsilon)$ is given by ${ }^{17}$

$$
f_{0}^{j}(\epsilon)=\frac{n_{0} C_{n}^{j}+e_{p}^{j}(\epsilon)}{R_{0}^{j}(\epsilon)},
$$

with the sum of capture and emission rates $R_{0}^{j}(\epsilon)=n_{0} C_{n}^{j}$ $+p_{0} C_{p}^{j}+e_{n}^{j}(\epsilon)+e_{p}^{j}(\epsilon)$.

It may be appropriate here to introduce quasi-Fermi levels for trapped electrons $\epsilon_{t n}^{j}$ and holes $\epsilon_{t p}^{j}$ defined by an equality

$$
R_{c}^{j} \equiv n_{0} C_{n}^{j}+p_{0} C_{p}^{j}=e_{n}^{j}\left(\epsilon_{t n}^{j}\right)=e_{p}^{j}\left(\epsilon_{t p}^{j}\right),
$$

for the energy-independent capture coefficients. The total transition rate $R_{0}^{j}(\epsilon)$ is approximated by $e_{n}^{j}(\epsilon)$ for energy above $\epsilon_{t n}^{j}, R_{c}^{j}$ between $\epsilon_{t n}^{j}$ and $\epsilon_{t p}^{j}$, and $e_{p}^{j}(\epsilon)$ below $\epsilon_{t p}^{j}$. The defect states of type $j$ are practically empty at $\epsilon>\epsilon_{t n}^{j}$ in the steady state $\left(f_{0}^{j}=0\right)$ due to a strong thermal emission of electrons, while at $\epsilon<\epsilon_{t p}^{j}$, a thermal emission of holes prevails so that the defect states are filled with electrons $\left(f_{0}^{j}=1\right)$. The fractional occupation $f_{0}^{j}=n_{0} C_{n}^{j} / R_{c}^{j}$ takes place between $\epsilon_{t n}^{j}$ and $\epsilon_{t p}^{j}$. Applying a regional approximation in energy to the mathematical treatment of the integrands involved in Eqs. (9)-(12) leads to

$$
\begin{aligned}
N= & \sum_{j} \int_{\epsilon_{t n}^{j}}^{\epsilon_{c}} d \epsilon C_{n}^{j} D^{j}(\epsilon) \frac{i \omega}{i \omega+e_{n}^{j}(\epsilon)} \\
& +\sum_{j} \int_{\epsilon_{t p}^{j}}^{\epsilon_{t n}^{j}} d \epsilon C_{n}^{j} D^{j}(\epsilon) \frac{p_{0} C_{p}^{j}}{R_{c}^{j}} \frac{i \omega}{i \omega+R_{c}^{j}}, \\
P= & \sum_{j} \int_{\epsilon_{v}}^{\epsilon_{t p}^{j}} d \epsilon C_{p}^{j} D^{j}(\epsilon) \frac{i \omega}{i \omega+e_{p}^{j}(\epsilon)} \\
& +\sum_{j} \int_{\epsilon_{t p}^{j}}^{\epsilon_{t n}^{j}} d \epsilon C_{p}^{j} D^{j}(\epsilon) \frac{n_{0} C_{n}^{j}}{R_{c}^{j}} \frac{i \omega}{i \omega+R_{c}^{j}}, \\
\bar{N}= & \sum_{j} \int_{\epsilon_{t p}^{j}}^{\epsilon_{t n}^{j}} d \epsilon C_{n}^{j} D^{j}(\epsilon) \frac{p_{0} C_{p}^{j}}{R_{c}^{j}} \frac{p_{0} C_{p}^{j}}{i \omega+R_{c}^{j}}, \\
\bar{P}= & \sum_{j} \int_{\epsilon_{t p}^{j}}^{\epsilon_{t n}^{j}} d \epsilon C_{p}^{j} D^{j}(\epsilon) \frac{n_{0} C_{n}^{j}}{R_{c}^{j}} \frac{n_{0} C_{n}^{j}}{i \omega+R_{c}^{j}} .
\end{aligned}
$$

\section{B. Emission- and capture-limited MPC responses}

A frequency-dependent MPC associated with emission and capture processes is manifested by Eqs. (14)-(17). The first terms in the expressions for $N$ and $P$ contain emissioncontrolled response functions $i \omega /\left(i \omega+e_{n, p}^{j}\right)$, whereas carrier capture processes are found to limit responses of the other terms. A capture-limited response also occurs for $\bar{N}$ and $\bar{P}$. The emission- and capture-limited responses alternate in determining the MPC behavior depending upon the relative magnitude of modulation frequency $\omega$ compared to the transition rate $R_{0}^{j}\left(\epsilon_{t n, p}^{j}\right)=2 R_{c}^{j}$. For the frequency $\omega$ larger than every $2 R_{c}^{j}$, the parameters $\bar{N}$ and $\bar{P}$ become negligibly small relative to $N$ and $P$ so that Eqs. (7) and (8) are reduced to

$$
\begin{aligned}
& \frac{n_{\omega}}{G_{\omega}}=\frac{1}{i \omega+N}, \\
& \frac{p_{\omega}}{G_{\omega}}=\frac{1}{i \omega+P} .
\end{aligned}
$$

The parameters $N$ and $P$ being simplified to

$$
\begin{aligned}
& N=\frac{1}{\tau_{n}}+\sum_{j} \int_{\epsilon_{t n}^{j}}^{\epsilon_{c}} d \epsilon C_{n}^{j} D^{j}(\epsilon) \frac{i \omega}{i \omega+e_{n}^{j}(\epsilon)}, \\
& P=\frac{1}{\tau_{p}}+\sum_{j} \int_{\epsilon_{v}}^{\epsilon_{t p}^{j}} d \epsilon C_{p}^{j} D^{j}(\epsilon) \frac{i \omega}{i \omega+e_{p}^{j}(\epsilon)},
\end{aligned}
$$


are indicative of a fully emission-limited MPC response. Lifetime constants $\tau_{n, p}$ appearing in the above equations are defined by

$$
\begin{aligned}
& \frac{1}{\tau_{n}}=\sum_{j} \int_{\epsilon_{t p}^{j}}^{\epsilon_{t n}^{j}} d \epsilon C_{n}^{j} D^{j}(\epsilon) \frac{p_{0} C_{p}^{j}}{R_{c}^{j}}, \\
& \frac{1}{\tau_{p}}=\sum_{j} \int_{\epsilon_{t p}^{j}}^{\epsilon_{t n}^{j}} d \epsilon C_{p}^{j} D^{j}(\epsilon) \frac{n_{0} C_{n}^{j}}{R_{c}^{j}} .
\end{aligned}
$$

When the opposite relation stands between $\omega$ and $2 R_{c}^{j}$, the emission-related terms involved in Eqs. (14) and (15) turn less dominant since $\omega \ll e_{n, p}^{j}(\epsilon)$ in the integrands, implying that MPC response becomes capture limited. The expressions for $n_{\omega}$ and $p_{\omega}$ are, if the frequency is low enough, found to be

$$
\begin{aligned}
& n_{\omega}=G_{\omega} \tau_{n}, \\
& p_{\omega}=G_{\omega} \tau_{p} .
\end{aligned}
$$

A further simplification is possible for the formulae derived in the emission-limited regime., ${ }^{5,79}$ Integrands in the expressions involve functions $i \omega /\left[i \omega+e_{n, p}^{j}(\epsilon)\right]$, the imaginary parts of which have a bell-shaped curve in energy $\epsilon$ and falling exponentially with a slope $k T$ on either side of $\epsilon=\epsilon_{\omega n, p}^{j}$, respectively. Here, $\epsilon_{\omega n, p}^{j}$ define the energies at which the emission rates $e_{n, p}^{j}(\epsilon)$ coincide with the frequency $\omega$. The peaked functions can be approximated to delta functions $(\pi k T / 2) \delta\left(\epsilon-\epsilon_{\omega n, p}^{j}\right)$ for a slowly varying distribution $D^{j}(\epsilon)$. The corresponding criterion is stated with the characteristic temperature

$$
T_{c}^{j}(\epsilon)=\left[k \frac{d \ln D^{j}(\epsilon)}{d \epsilon}\right]^{-1}
$$

as $T<\left|T_{c}^{j}\left(\epsilon_{\omega n, p}^{j}\right)\right|$. Applying the approximation, for electron concentration $n_{\omega}$ one obtains

$$
\operatorname{Im}\left(\frac{G_{\omega}}{n_{\omega}}\right)=\omega+\frac{\pi k T}{2} \sum_{j} C_{n}^{j} D^{j}\left(\epsilon_{\omega n}^{j}\right)
$$

with

$$
\epsilon_{\omega n}^{j}=\epsilon_{c}-k T \ln \left(\frac{N_{c} C_{n}^{j}}{\omega}\right)
$$

and for hole concentration $p_{\omega}$

$$
\operatorname{Im}\left(\frac{G_{\omega}}{p_{\omega}}\right)=\omega+\frac{\pi k T}{2} \sum_{j} C_{p}^{j} D^{j}\left(\epsilon_{\omega p}^{j}\right)
$$

with

$$
\epsilon_{\omega p}^{j}=\omega_{v}+k T \ln \left(\frac{N_{v} C_{p}^{j}}{\omega}\right)
$$

The above equations suggest two capabilities of MPC measurements to profile the density of states $D^{j}\left(\epsilon_{\omega n, p}^{j}\right)$ by scanning frequency $\omega$ at fixed temperature $T$ and by scanning temperature $T$ at fixed frequency $\omega$. It is obvious that the spectroscopic techniques essentially lose their applicability in the capture-limited regime where MPC tends to be inphase, as found by Eqs. (22) and (23), and the quantities

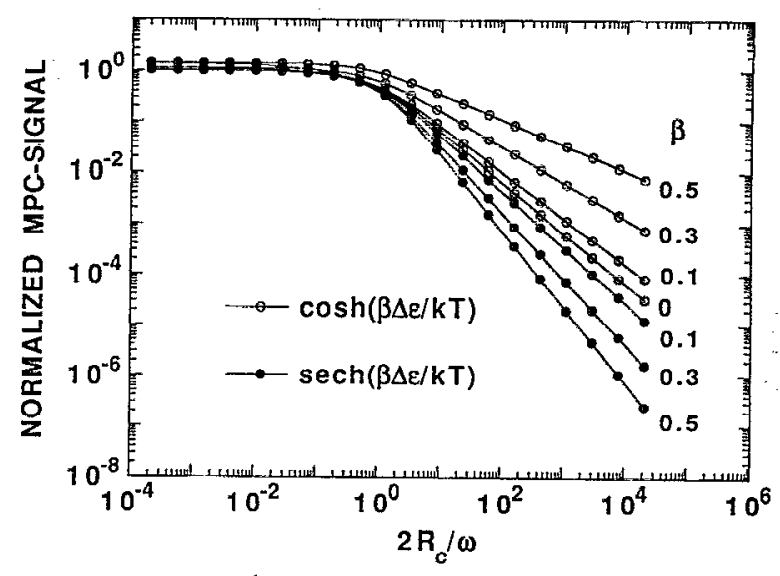

FIG. 1. Plots of the normalized MPC signal $S / C_{n} D\left(\epsilon_{\omega n}\right)$ vs $2 R_{c} / \omega$ for the defect distributions such that $D(\epsilon) \propto \cosh (\beta \Delta \epsilon / k T)$ (O) and $D(\epsilon) \propto \operatorname{sech}(\beta \Delta \epsilon / k T)(\bigcirc)$ with $\Delta \epsilon=\epsilon_{c}-\epsilon_{g} / 2 . k T=25 \mathrm{meV}, \epsilon_{g}=1.8 \mathrm{eV}$, $\epsilon_{F}=\epsilon_{c}-\epsilon_{g} / 2, N_{c, v}=10^{20} \mathrm{~cm}^{-3}, C_{n, p}=10^{-8} \mathrm{~cm}^{3} \mathrm{~s}^{-1}$, and $\epsilon_{\omega n}=\epsilon_{c}-0.6$ $\mathrm{eV}$ are chosen in the calculation. The parameter $\beta$ is taken as $0,0.1,0.3$, and 0.5 .

$\operatorname{Im}\left(G_{\omega} / n_{\omega}\right)$ and $\operatorname{Im}\left(G_{\omega} / p_{\omega}\right)$ vanish and become irrelevant to $D^{j}\left(\epsilon_{\omega n, p}^{j}\right)$. In Sec, II C, the transition between the emissionand the capture-limited MPC responses will be further discussed in detail.

\section{Evolution of MPC response with illumination level}

In this section, for the sake of simplicity, we assume that electrons are the predominant type of carriers in photoconduction so that $\sigma_{0, \omega}=e \mu_{n} n_{0, \omega}$. In this case, as found by Eq. (24), the MPC signal defined by

$$
S=-\frac{2}{\pi k T}\left\{\frac{e \mu_{n} G_{\omega}}{\left|\sigma_{\omega}\right|} \sin \left[\operatorname{Arg}\left(\sigma_{\omega}\right)\right]+\omega\right\},
$$

yields the summation of the density of states $D^{j}\left(\epsilon_{\omega n}^{j}\right)$ multiplicd by the capture coefficient $C_{n}^{j}$ in the emission-limited regime. The term $\omega$ in parens is normally negligible. On the other hand, by increasing the capture rate far beyond the modulation frequency, MPC behavior turns capture limited. In this regime, the phase shift that tends towards zero causes a decreasing signal magnitude. The transition between two regimes can be found in the experiment that varies the illumination level $G_{0}$ at fixed frequency and fixed temperature.

In order to study the transition region as well as to gain an insight into illumination-evolution experiments, we have numerically computed the MPC signal $S$ from the basic Eqs. (7)-(12) for various distributions $D^{i}(\epsilon)$. The steady-state carrier concentrations $n_{0}$ and $p_{0}$ were determined from the charge neutrality for the assumed distribution $D^{j}(\epsilon)$. The dark equilibrium Fermi level $\epsilon_{F}$ lying at the center of the gap $\epsilon_{g}=1.8 \mathrm{eV}$ and the identical capture coefficients $C_{n}^{j}=C_{p}^{j}$ were chosen for the calculations.

To start with, we shall discuss the case when there are defects of a single type. (The superscript $j$ is omitted in the following description.) Figure 1 presents the results obtained for $\epsilon_{\omega n}=\epsilon_{c}-0.6 \mathrm{eV}$ with distributions $D(\epsilon)$ varying as $\cosh (\beta \Delta \epsilon / k T)$ and as $\operatorname{sech}(\beta \Delta \epsilon / k T)$, where $\Delta \epsilon$ denotes the energy separation from the center of the gap. The trend seen 


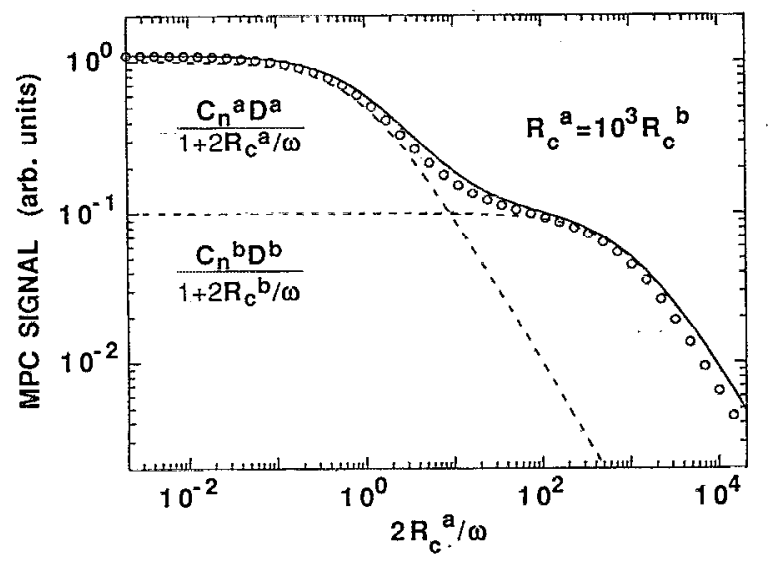

FIG. 2. MPC signal $S$ for uniform defect distributions $D^{j}(\epsilon)=D^{j} \quad(j=a$ and $b$ ) as a function of the ratio $2 R_{c}^{a} / \omega$. In the calculation, $k T=25 \mathrm{meV}$, $\epsilon_{\mathrm{g}}=1.8 \mathrm{eV}, \epsilon_{F}=\epsilon_{\mathrm{c}}-\epsilon_{\mathrm{g}} / 2, \quad N_{c, v}=10^{20} \mathrm{~cm}^{-3}, C_{n, p}^{a}=10^{-6} \mathrm{~cm}^{3} \mathrm{~s}^{-1}$, $C_{n, p}^{b}=10^{-0} \mathrm{~cm}^{3} \mathrm{~s}^{-1}, C_{n}^{a} D^{a} / C_{n}^{b} D^{b}=10$, and $\epsilon_{\omega n}^{a}=\epsilon_{\mathrm{c}}-0.6 \mathrm{eV}$ are chosen. Solid curve is the plot calculated from Eq. (27).

in the figure is in good accordance with that mentioned earlier; the MPC signal $S$ stays at $C_{n} D\left(\epsilon_{\omega n}\right)$ when $2 R_{c} \ll \omega$, while the curve is dropped for a larger $2 R_{c} / \omega$ ratio. It is also found that the signal decrease follows approximately $R_{c}^{\alpha-1}$, where the exponent $\alpha$ corresponds to the ratio of the temperature $T$ to $T_{c}\left(\epsilon_{\omega n}\right)$, that is, $\alpha=\beta>0$ for $\cosh (\beta \Delta \epsilon / k T)$ and $\alpha=-\beta<0$ for $\operatorname{sech}(\beta \Delta \epsilon / k T)$. The calculations have been also carried out for more structured $D(\epsilon)$ as well as for the different $\epsilon_{\omega n}$ positions. It was confirmed from the results that the variations of $S$ with $R_{c}$ around the transition region can be well represented by a simple relation that $S=C_{n} D\left(\epsilon_{\omega n}\right) /\left(1+2 \mathrm{~K}_{\mathrm{c}} / \omega\right)^{\mathrm{I}-\alpha}$ with $\alpha=T / T_{c}\left(\epsilon_{\omega n}\right)$. Note that the $\alpha$ value around zero is consistent with the present MPC theory assuming that $T<\left|T_{c}\left(\epsilon_{\omega n}\right)\right|$.

The simulation study has been extended to the MPC response for defects composed of the different species $j$ with the capture coefficients $C_{n, p}^{j}$. Figure 2 illustrates - the illumination-evolution curve calculated for two types $j=a$ and $j=b$ of the defect states distributed uniformly in energy; $D^{j}(\epsilon)=D^{j}$. Readily seen in the figure is that the curve exhibits a staircase-like behavior. Each step of the magnitude $C_{n}^{j} D^{j}$ arises from the transition between the emission- and the capture-limited regimes at $2 R_{c}^{j}=\omega$. The results presented here convince us that a general expression is written as

$S=\sum_{j} C_{n}^{j} D^{j}\left(\epsilon_{\omega n}^{j}\right)\left[\frac{1}{1+2 R_{c}^{j} / \omega}\right]^{1-\alpha^{j}}$ with $\alpha^{j}=\frac{T}{T_{c}^{j}\left(e_{\omega n}^{j}\right)}$.

A significant suggestion from our simulation is the possibility of simultaneously determining $C_{n}^{j} D^{j}\left(\epsilon_{\omega n}^{j}\right)$ and $R_{c}^{j}$ from the illumination-evolution data. If the capture rate of one type of carrier is predominant, and if the same type of carrier has a major contribution to the conductivity $\sigma_{0}$, then the corresponding capture coefficient can be determined by the measurement of the rate $R_{c}^{j}$. For $\sigma_{0}=e \mu_{n} n_{0}$ that we assume in this section, the absolute estimate of the electron capture coefficient $C_{n}^{j}$ can be obtained when $R_{c}^{j} \cong n_{0} C_{n}^{j} \gg p_{0} C_{p}^{j}$ and permits us to translate a given $\omega$ and
$T$ to $\epsilon_{\omega n}^{j}$ as well as a derived $C_{n}^{j} D^{j}\left(\epsilon_{\omega n}^{j}\right)$ to $D^{j}\left(\epsilon_{\omega n}^{j}\right)$. Although the ratios $C_{n}^{j} / C_{p}^{j}$ are not known a priori, the relation that $n_{0} C_{n}^{j} \gg p_{0} C_{p}^{j}$ may be trusted for $n_{0} \gg p_{0}$. The charge neutrality condition implies that the larger $n_{0}$ is likely to occur in the case where the density of states between $\epsilon_{t n}^{j}$ and $\epsilon_{F}$, by which excess electrons can be captured, is sufficiently smaller than the density of the states between $\epsilon_{F}$ and $\epsilon_{t p}^{j}$, by which excess holes can be captured. Knowledge of the $\epsilon_{F}$ location, closely linked with the ratio $n_{0} / p_{0}$, is thus an important prerequisite for the experimental analysis.

Finally, we must comment on the applicability of Eq. (27) obtained by assuming the occupation statistics for monovalent defect states to the case where divalent dangling bond states are present in the material. Detailed theoretical studies of the influence of the dangling bond states on MPC at a fixed illumination level have been recently made by Longeaud and Kleider. ${ }^{12}$ It was proved in their literature that MPC related to a single distribution $D(\epsilon)$ of dangling bond states with a positive correlation energy $U$ can be approximately represented as two distributions $D(\epsilon)$ and $D(\epsilon+U)$ of monovalent states belonging to different trap species. On the basis of their analytical results, we further discuss the MPC evolution with the illumination level for positively correlated states in Appendix A, where a direct extension of Eq. (27) is justified.

\section{MPC SPECTROSCOPY MEASUREMENTS ON UNDOPED AMORPHOUS SILICON}

\section{A. Experimental details}

The sample studied in this work was an undoped $\mathrm{rf}$ glow-discharged $\dot{a}$-Si:H film deposited on a glass substrate. The substrate temperature during the growth was $300^{\circ} \mathrm{C}$ and the film thickness was $1.5 \mu \mathrm{m}$. Coplanar $\mathrm{Al}$ electrodes 15 $\mathrm{mm}$ long were evaporated on the top of the film, with the electrode spacing being $1 \mathrm{~mm}$. Prior to the MPC measurements the sample was annealed at $200^{\circ} \mathrm{C}$ in vacuum to remove all light-induced defects.

The Fermi-level position of the sample was determined to be $\epsilon_{F}=\epsilon_{c}-0.7 \mathrm{eV}$ from the room-temperature dark conductivity using the pre-exponential factor $150 \Omega^{-1} \mathrm{~cm}^{-1}, 18$ and the optical bang gap was measured to be $1.75 \mathrm{eV}$. The Fermi level lying closer to the conduction band implies that majority photocarriers are electrons $\left(n_{0} \gg p_{0}\right)$, in accordance with the charge neutrality argument in Sec. II C and, therefore, lends credence to the predominant electron capture rate, that is, $R_{c}^{j} \cong n_{0} C_{n}^{j}$. Since the mobility of electrons $\left(\mu_{n}=10\right.$ $\mathrm{cm}^{2} / \mathrm{V} \mathrm{s}$ ), approximately ten times larger than that of holes, is suggested experimentally, ${ }^{19}$ assuming the unipolar photoconduction carried by electrons such that $\sigma_{n, \omega}=e \mu_{n} n_{0, \omega}$ is appropriate to the experimental analysis.

In the MPC experiments, the sample was mounted in an evacuated, temperature-controlled optical cryostat. The measurement temperature was varied between 140 and $320 \mathrm{~K}$. A light-emitting diodc with pcak fluxes at $655 \mathrm{~nm}$ was used to uniformly illuminate the sample. The light intensity was modulated at a frequency of $\omega=2 \pi f$ with $f$ varying between $0.63 \mathrm{~Hz}$ and $1 \mathrm{kHz}$. The amplitude and phase shift of the 


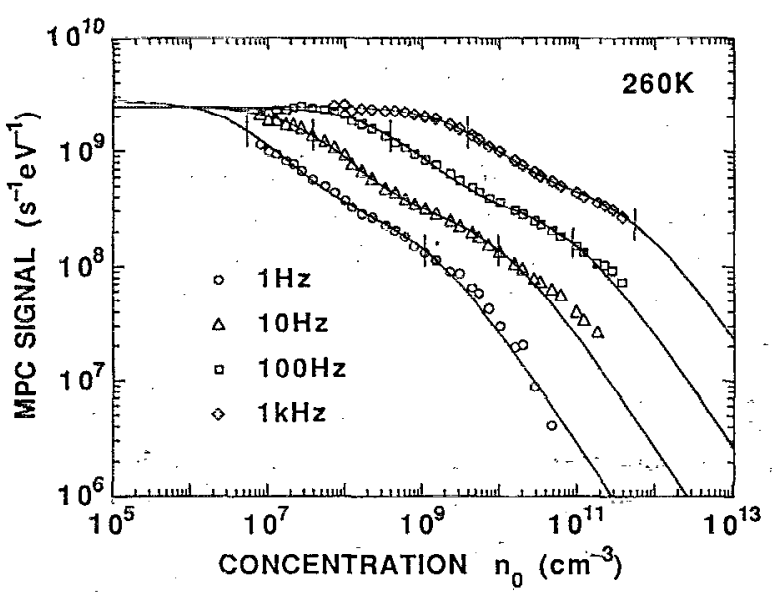

FIG. 3. Illumination evolution of MPC signal measured at temperature $T=260 \mathrm{~K}$ for various frequencies $f=1,10,100$, and $1 \mathrm{kHz}$. Solid curves are fits to the data calculated from Eq. (27) with $\alpha^{j}=0$. Vertical bars indicate the positions of $n_{0}=\omega / 2 C_{n}^{j}$.

resulting MPC were measured with a lock-in amplifier and a low-noise high gain current amplifier.

\section{B. Capture coefficient and density of defect states}

The results of illumination-evolution measurements at $T=260 \mathrm{~K}$ are presented in Fig. 3, where the MPC signals $S$ obtained at various frequencies $f$ are plotted against the electron concentration $n_{0}$ determined from the steady-state conductivity $\sigma_{0}$. It is found in the figure that $S$ curves show a plateau in the lower $n_{0}$ region, while in the higher $n_{0}$ region, they go downward with $n_{0}$. Also found is the fact that each curve shifts by increasing the frequency $f$ toward the higher $n_{0}$ side. These features are consistent with those predicted in Sec. $\Pi \mathrm{C}$. The best fits calculated from the characteristics (27) assuming two kinds of defect states with $\alpha^{j}=0$ are plotted by solid lines; the vertical bars indicate the positions of $n_{0}=\omega / 2 C_{n}^{j}$. The capture coefficients $C_{n}^{j}$ estimated from the illumination-evolution data are summarized in Fig. 4 as a function of the measurement temperature $T$. Both coefficients are found to be almost temperature independent and

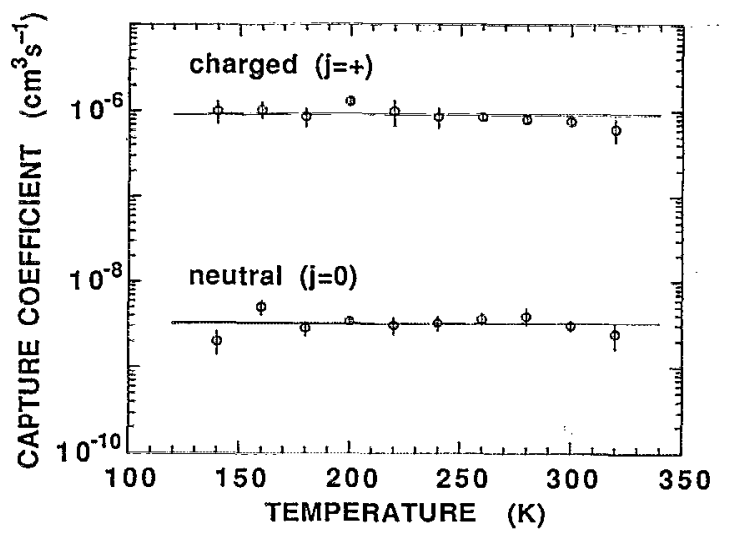

FIG. 4. Temperature dpendences of the mcasured capture coefficients.

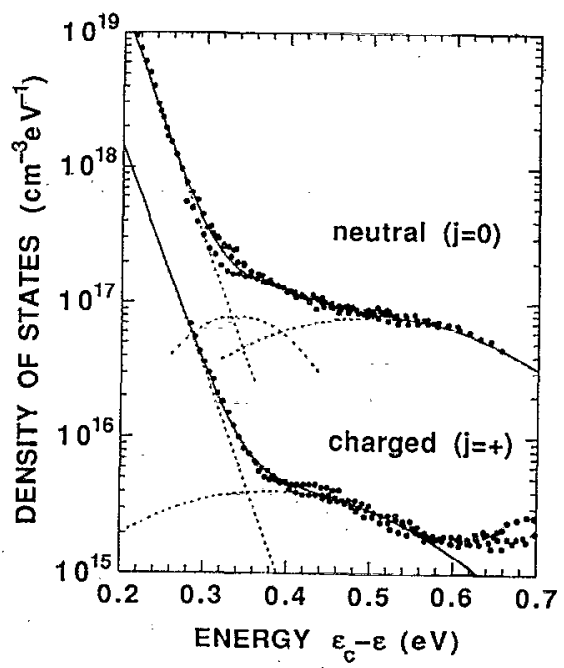

FIG. 5. Density-of-states spectra obtained from the MPC measurements. Solid curves represent fits to the data assuming exponential and Gaussian distributions (dotted lines).

are kept at around $9 \times 10^{-7}$ and $3 \times 10^{-9} \mathrm{~cm}^{3} \mathrm{~s}^{-1}$, respectively. The considerable difference between these magnitudes might be attributed to difference in the charge states of defects. Here we shall assign the defect with the larger coefficient to being positively charged $(j=+)$, and the defect with the smaller coefficient to being neutral $(j=0)$. The validity of these designations will be discussed in detail in Sec. IV A.

Figure 5 displays the defect distributions $D^{j}(\epsilon)$ deduced from the MPC data using the measured $C_{n}^{j}$ values and assuming $N_{c}=10^{20} \mathrm{~cm}^{-3}$. The spectra for both the $j=+$ and $j=0$ defect components appear to be comprised of a band tail superimposed on a broad bump lying at $0.4-0.5 \mathrm{eV}$ below the conduction band edge $\epsilon_{c}$. Exponential and Gaussian distributions were assumed for a fit to the raw spectra, the results of which are shown in the figure. For the $j=+$ component, the Gaussian distribution is centered at $\epsilon_{0}=\epsilon_{c}-0.38 \mathrm{eV}$ with a standard deviation $s=150 \mathrm{meV}$ and an integrated density of $N_{\text {int }}=1.5 \times 10^{15} \mathrm{~cm}^{-3}$. On the other hand, a better fit to the data for the $j=0$ component needs two Gaussian distributions with $\epsilon_{0}=\epsilon_{c}-0.5 \mathrm{eV}$, $s=150 \mathrm{meV}$ and $N_{\text {int }}=2.9 \times 10^{16} \mathrm{~cm}^{-3}$, and with $\epsilon_{0}=\epsilon_{c}-0.34 \mathrm{eV}, s=70 \mathrm{meV}$ and $N_{\text {int }}=1.4 \times 10^{16} \mathrm{~cm}^{-3}$. The existence of the narrow defect band at the shallow energy position $\left(\epsilon_{0}=\epsilon_{\mathrm{c}}-0.34 \mathrm{eV}, s=70 \mathrm{meV}\right)$ agrees well with a previous report. ${ }^{10}$ in which its origin is inferred to be any structural defects formed at an $a-\mathrm{Si}: \mathrm{H} /$ glass interface. We suggest that the deep and broad defect bands $\left(\epsilon_{0}=\epsilon_{c}-0.38\right.$ $\mathrm{eV}$ and $\epsilon_{0}=\epsilon_{c}-0.5 \mathrm{eV} ; s=150 \mathrm{meV}$ ) originate from Si dangling bonds that exist in the bulk region. An observed concurrence of charged and neutral dangling bonds in undoped $a-\mathrm{Si}: \mathrm{H}$ will be quantitatively discussed according to a thermodynamical defect formation model in Sec. IV B.

The exponent $\alpha^{j}$ applied to the analysis of the illumination-evolution data should be taken to correspond with an energy gradient of the distribution $D^{j}(\epsilon)$ derived from it. The relatively slowly varying distribution of the defect bands agrees with the assumption that $\alpha^{j}=T / T_{c}^{j}=0$. 
The same extent of accuracy may not hold, however, for the exponential distributions of which characteristic temperature $T_{c} \cong 300 \mathrm{~K}$ is estimated since the measurement temperatures $T=140-160 \mathrm{~K}$ at which MPC arising from the tail states is detected give the temperature ratio $T / T_{c} \cong 0.5$. We have tested the accuracy by applying the $\alpha^{j}$ values ranging from 0 to 0.5 to the determination of $C_{n}^{j}$ and $D^{j}(\epsilon)$. This confirmed that varying $\alpha^{j}$ values does not significantly affect the results, and that two exponential tails with $T_{c} \cong 300 \mathrm{~K}$ are not artifacts of that particular assumption. Most peculiar in the present experimental results is the-different types of tail states. The interpretation for the capture coefficients relying on the differing charge states cannot directly apply to the conduction band tail states since their positively charged state is hardly likely. In Sec. IV A, we will address the physical implications of the observation as well as discuss the capture processes for charged and neutral defects.

Before concluding this section, we will compare the defect distribution derived here to those reported previously. ${ }^{6,8,10,11}$ The previous MPC measurements have normally been performed at fixed light intensity, assuming, $a$ priori, a fully emission-limited MPC response. Such assessments of defect distribution are not generally justifiable, as was demonstrated by our illumination-evolution experiments. Moreover, possible defect components remain unresolved by the previous studies. Any quantitative comparison is impossible, therefore, we restrict our attention to the overall feature seen in the spectra. The less-structured distribution concluded here seems to be in reasonable accord with the MPC spectra observed in the previous work using a coplanar configuration. ${ }^{8.10}$ From the measurements of modulated primary-photocurrent $J_{\omega}$ in a time-of-flight (TOF) configuration, ${ }^{6,11}$ on the other hand, a sharply peaked distribution around the midgap has been evaluated according to a relation that

$$
C_{n} D\left(\epsilon_{\omega n}^{-}\right)=-\frac{2}{\pi k T}\left\{\frac{e \mu_{n} E G_{\omega}}{\left|J_{\omega}\right|} \sin \left[\operatorname{Arg}\left(J_{\omega}\right)\right]+\omega\right\},
$$

where $E$ denotes the electric field. By replacing $J_{\omega} / E$ with $\sigma_{\omega}$, the right-hand side of the equation coincides with our definition of the MPC signal [Eq. (26)], which is derived for a secondary-photocurrent case. The theoretical consideration of TOF experiments under a modulated illumination is presented in Appendix B, where it is proved that their analysis is validated only for a low field case when the electron transit time $t_{\text {transit }}$ is much longer than the trapping time $t_{\text {trap }}$ defined by Eq. (B5). However, it can be seen from their experimental data that $t_{\text {transit }}$ is shorter than or comparable to $t_{\text {trap }}$ in the measurements of the midgap defects. Applying the theoretical analysis adjusted for modulated photocurrent measurements might reconcile the discrepancy in the reconstructed defect distribution; this however, is beyond the focus of this work.

\section{DISCUSSION}

\section{A. Carrier capture processes for neutral and charged defects}

Assuming a spatially uniform current flow of ballistic electrons having a thermal velocity $v_{\text {th }}$ into the neighborhood of a defect center where the capture can occur, the capture coefficient $C_{n}$ is generally written with the capture cross section $\sigma_{n}$ as

$$
C_{n}=v_{\mathrm{th}} \sigma_{n}
$$

This description for the capture process is reasonably verified by high mobility solids, where the electron mean free path $l_{n}$ is sufficiently longer than the capture radius $r_{n}=\sqrt{\sigma_{n} / \pi}$, and the rapid electron delivery sustains the Boltzmann concentration near the center. A simple expression for the capture cross section can be derived for the defect center with a Coulomb attractive potential. According to a classical Thomson approach, ${ }^{20}$ one obtains

$$
\sigma_{n}=\frac{4 \pi}{3} \frac{r_{c}^{3}}{l_{n}}
$$

where $r_{c}=e^{2} / 4 \pi \kappa k T$ is the Coulomb radius, and $\kappa$ the dielectric constant of the material. The radius $r_{c}$ defines a sphere in the interior of which the Coulomb binding energy cxcceds the thermal energy, indicating that any thermal escape of an electron from the center is hardly probable.

As low mobility solids, where the opposite relation that $r_{n}>l_{n}$ holds are concerned, the rate at which electrons are delivered is low relative to the rate at which the center can capture so the center's neighborhood is depleted of electrons. Electron diffusion toward the depletion area near the center is then likely to limit the caplure process. In such a situation, the capture coefficient is expressed with the diffusion constant $D_{n}=k T \mu_{n} / e$ in the form ${ }^{21}$

$$
C_{n}=4 \pi D_{n} r_{n} \text {. }
$$

Replacing the capture radius $r_{n}$ by the Coulomb radius $r_{c}$ yields the Langevin formula for the Coulomb attractive center. $^{22}$

$$
C_{n}=\frac{e \mu_{n}}{\kappa} .
$$

In accordance with the ballistic and diffusive models for electron capture, we will discuss the experimental results for the capture coefficients. For the smaller capture coefficient $C_{n}^{0}=3 \times 10^{-9} \mathrm{~cm}^{3} / \mathrm{s}$, the capture radius around room temperature is estimated as $r_{n} \cong 1 \AA$ for the ballistic model and as $r_{n} \cong 0.1 \AA$ for the diffusive model. The mean free path of an electron $l_{n} \cong 10 \AA$ suggested for $a$-Si:H materials ${ }^{23-25}$ clearly indicates that the ballistic model is certainly applicable for $C_{n}^{0}$. On the other hand, a capture radius slightly larger than the mean free path is estimated for the larger coefficient $C_{n}^{+}=9 \times 10^{-7} \mathrm{~cm}^{3} / \mathrm{s}$. For the charged defect, a clearer distinction between the diffusive and ballistic processes lies in the temperature dependence of the capture coefficient. Theoretically, the capture coefficient following the Langevin formula depends only on the mobility, whereas the Thomson formula involving the Coulomb radius predicts a 
stronger variation with temperature. An invisible temperature dependence experimentally observed for $C_{n}^{+}$is considered to be consistent with the diffusive model. Application of the Langevin formula leads to a theoretical value $1.5 \times 10^{-6}$ $\mathrm{cm}^{3} / \mathrm{s}$ using $\kappa=12 \kappa_{0}$, which is in good agreement with the measured value and therefore gives confirmation to our designation.

The previous discussion of the capture processes supposes that the principal capture orbits of neighboring centers are not overlapped. A negligibly weak reciprocal influence on the deep defects of the density $10^{15}-10^{16} \mathrm{~cm}^{-3}$ is reasonably assessed from the long average intercenter distance $300-600 \AA$. In the presence of the tail states of the density on the order of $10^{20} \mathrm{~cm}^{-3}, 19$ however, it is probable that a fraction of the tail states lies in close vicinity to the deep defect, and that the defect state and the intimate tail states participate in a carrier capture event cooperatively. If such a cluster of capture centers is composed of the charged defect surrounded by the tail states, electrons pulled by the defectinducing Coulomb attraction would be fed to the tail states as a certain probability. The capture efficiency for the particular tail states is then enhanced substantially and distinguished from that for isolated states. A qualitative explanation for the present experimental observation can thus be obtained by assuming the existence of a defect cluster although further studies concerned with the electronic process are clearly needed.

\section{B. Defect formation in undoped amorphous silicon}

We base the discussion here on the defect formation model ${ }^{16}$ assuming a thermodynamical equilibration process where dangling bond defects are created by the breaking of weak $\mathrm{Si}-\mathrm{Si}$ bonds, which are generally thought to be associated with the valence band tail states distributed like $\exp \left[-\left(\epsilon-\epsilon_{v}\right) / k T_{v}\right]$. The defect formation may be stabilized by a diffusive hydrogen motion through breaking and reforming $\mathrm{Si}-\mathrm{H}$ bonds. When a distribution of energies at which a defect could form is present due to the inherent disorder of the amorphous network, the resulting equilibrium-defect distribution consists of three separated components formed as positively charged dangling bonds $\left(D^{+}\right.$states), neutral dangling bonds ( $D^{0}$ states), and negatively charged dangling bonds ( $D^{-}$states). The density of states $D(\epsilon)=D^{+}(\epsilon)+D^{0}(\epsilon)+D^{-}(\epsilon)$ is frozen-in below the equilibration temperature $T_{\mathrm{eq}}$. Adopting notations $D_{h, z, e}$ instead of $D^{+, 0,-}$ is useful ${ }^{16}$ since we can clearly distinguish between contributions to $D(\epsilon)$ from the $+/ 0$ and $0 /-$ oneelectron transition levels separated by the effective correlation energy $U$. The $+/ 0$ transition levels for $D_{h, z, e}$ are peaked, respectively, at energies

$$
\begin{aligned}
& \epsilon_{h}^{+/ 0} \cong \epsilon_{p}, \\
& \epsilon_{z}^{+10} \cong \epsilon_{p}-\frac{s_{p}^{2}}{k T^{*}}, \\
& \epsilon_{e}^{+10} \cong \epsilon_{p}-\frac{2 s_{p}^{2}}{k T^{*}},
\end{aligned}
$$

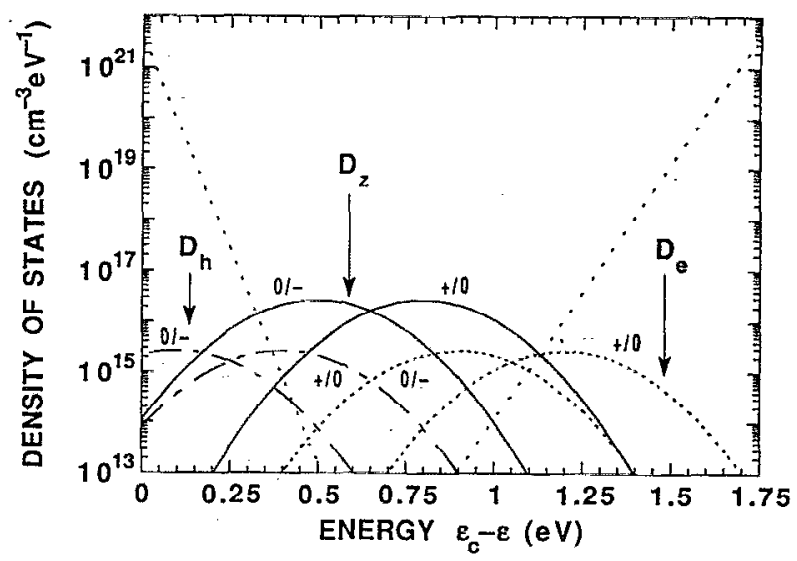

FIG. 6. Model of defect distribution in undoped $a$-Si:H.

for the pool of potential defects represented by a gaussian distribution with a maximum at energy $\epsilon_{p}$ and standard deviation $s_{p}{ }^{16}$ The temperature $T^{*}$ is defined by $T_{v}+m T_{\text {eq }} / 2$, where $m$ denotes the number of $\mathrm{Si}-\mathrm{H}$ bonds mediating the weak-bond breaking reaction.

Taking into account the dark defect occupation, the defect bands measured from low-illumination MPC are identified as due to the $+/ 0$ transition levels for the positively charged $D_{h}$ states and the $0 /-$ transition levels for the neutral $D_{z}$ states. If this interpretation is correct, the transition energies $\epsilon_{h}^{+/ 0} \cong \epsilon_{c}-0.4 \mathrm{eV}$ and $\epsilon_{z}^{0 /-} \cong \epsilon_{c}-0.5 \mathrm{eV}$ as well as the pool width $s_{p} \cong 150 \mathrm{meV}$ are readily assessed. The present estimate for $\epsilon_{h}^{+/ 0}$ is in excellent agreement with those from subband-gap optical absorption spectra ${ }^{14}$ and from thinfilm transistor characteristics. ${ }^{2}$ The transition energy $\epsilon_{e}^{0 /-} \cong \epsilon_{c}-0.9 \mathrm{eV}$ evaluated from JCT spectroscopy ${ }^{4}$ is generally accepted at present. Combining these values for transition energies leads to the estimate that $U \cong 0.3 \mathrm{eV}$ and $k T^{*} \cong 60 \mathrm{meV}$, both of which are consistent with the results of experimental analyses for the temperature dependence of spin-active neutral defect density in undoped $a-\mathrm{Si}: \mathrm{H} ;{ }^{26}$ $U=0.2-0.3 \mathrm{eV}$, and for the Fermi-level dependence of charged defect density in doped $a-\mathrm{Si}: \mathrm{H}^{13,14,27} k T_{*}^{*}=40-70$ $\mathrm{meV}$. The equilibrium Fermi level lying at $\epsilon_{F}=\left(\epsilon_{h}^{+/ 0}+\epsilon_{e}^{0 /-}\right) / 2$ gives an equal number of the charged states $D_{h}$ and $D_{e}$, and the charge neutrality for the intrinsic materials is maintained. ${ }^{16}$ Using the quantities mentioned, the Fermi-level position is calculated to be $\epsilon_{F}=\epsilon_{c}-0.65$ $\mathrm{eV}$, which differs by only $50 \mathrm{meV}$ from our experimental value. It is thus possible to account for, with reasonable accuracy, the data on the defect level distribution in a selfconsistent way in terms of the thermal equilibrium idea. The density of states suggested from this study is pictured in Fig. 6.

The pool width and the effective correlation energy substantally influence the equilibrium densities of charged and neutral dangling bonds for the intrinsic materials. According to the current defect pool model ${ }_{1}{ }^{16}$ the neutral dangling bond formation prevails when $U \gg s_{p}^{2} / k T^{*}$, while the charged dangling bonds outnumber the neutral dangling bonds when $U \ll s_{p}^{2} / k T^{*}$. The above = estimation that gives 
$s_{n}^{2} / k T^{*}-U \cong k T^{*}$ suggests comparable densities of charged and neutral dangling bonds. The theoretical prediction obviously contradicts with present experimental observation that $D_{z}$ density is roughly one order of magnitude larger than $D_{h}$ density. It is worthwhile to note that such a dominance of ncutral defects is also implied from other MPC works ${ }^{8,10}$ if their data are reinterpreted by applying our estimate of capture coefficients. They have assumed that measured MPC is related to deep defects with $C_{n}=10^{-8} \mathrm{~cm}^{3} \mathrm{~s}^{-1}$ and have evaluated the density to be $10^{15}-10^{16} \mathrm{~cm}^{-3}$. It can be said from the present work that a low-llumination MPC in undoped $a$-Si:H is likely to be dominated by the charged defects with a larger coefficient $C_{n}^{+} \cong 10^{-6} \mathrm{~cm}^{3} \mathrm{~s}^{-1}$, suggesting a severe overestimate of defect density in the previous works. This leads us to once again conclude that the density of charged defects is much smaller than that of neutral defects, which is known to be usually $10^{15}-10^{16} \mathrm{~cm}^{-3}$ from electron-spin-resonance measurements.

A physical explanation for the disagreement between the experimental result and the defect pool prediction is not clear at present and is the subject of further investigations. The current defect-pool description of equilibrium defect density as well as defect distribution relies on an assumption that the formation energy for the weak bond to dangling bond conversion can be represented by the one-electron energy difference of these states ${ }^{13,15,16}$ The present MPC observation might imply that some nonelectronic corrections are needed, as was suggested in a previous study of charged defect density of doped $a-\mathrm{Si}: \mathrm{H}^{11}$

\section{SUMMARY}

A theoretical analysis of MPC has been developed for a semiconductor containing different species of defects, and a novel experimental technique consisting of MPC measurements with a varying illumination level has been applied with good success to determine the energy distribution as well as the capture coefficients of defect states in undoped $a$-Si:H. The experiments revealed the capture-coefficientresolved defect distribution over a wide energy range from 0.2 to $0.7 \mathrm{eV}$ below the conduction band edge, in which a Gaussian-sbaped deep-defect band and an exponential band tail are identified. The experimental findings that follow lead us to gain some physical insight into the capture process as well as into defect formation.

(1) The measured electron capture coefficients are almost temperature independent. Their magnitudes $9 \times 10^{-7}$ and $3 \times 10^{9} \mathrm{~cm}^{3} / \mathrm{s}$ are consistent both with the diffusive capture by the positively charged defects and the ballistic capture by the neutral defects, respectively.

(2) The difference in the capture coefficients for conduction band tail states suggests a reciprocal influence of the capture centers. For a large density of the tail states, a finite number of them are built in close to the charged defect and exposed to the Coulomb attractive potential produced by the charged center. The enhanced capture efficiency distinguishes the intimate from the isolated tail states.

(3) The defect bands located around $\epsilon_{c}-0.4 \mathrm{eV}$ and $\epsilon_{c}-0.5 \mathrm{eV}$ are identified as originating from the positively charged $D^{+}$and the neutral $D^{0}$ dangling bonds, respectively.
The suggested energy placement of the dangling bond defects concludes a positive correlation energy $U=0.3 \mathrm{eV}$ according to the defect pool model.

(4) In the framework of the current model, the appearance of neutral dangling bonds in excess of charged dangling bonds assessed from the present experiment is quite unexpected. This observation might indicate the possibility that some nonelectronic effects are involved in the defect formation processes.

\section{APPENDIX A: MPC ASSOCIATED WITH POSITIVELY CORRELATED DEFECTS}

The behavior of MPC related to the presence of divalent dangling bond states with a correlation energy $U$ being either positive or negative has been theoretically studied by Longeaud and Kleider. ${ }^{12}$ It was shown from their analysis that a distribution of positively correlated states can roughly behave as two distributions of monovalent states: one distribution associated with $+/ 0$ transitions $D^{+/ 0}(\epsilon)$ with capture rates $n C_{n}^{+}$and $p C_{p}^{0}$ and emission rates $e_{n}^{0}(\epsilon)$ and $e_{p}^{+}(\epsilon)$, and another distribution associated with $0 /-$ transitions $D^{0 /-}(\epsilon)=D^{+/ 0}(\epsilon-U)$ with capture rates $n C_{n}^{0}$ and $p C_{p}^{-}$and emission rates $e_{n}^{-}(\epsilon)$ and $e_{p}^{0}(\epsilon)$. Specifically, when majority photocarriers are electrons $\left(n_{0} \gg p_{0}\right)$, the MPC signal defined by Eq. (26) can be represented approximately as

$$
S \cong C_{n}^{+} D^{+/ 0}\left(\epsilon_{\omega n}^{+/ 0}\right)+C_{n}^{0} D^{0 /-}\left(\epsilon_{\omega n}^{0 /-}\right),
$$

with energies $\epsilon_{\omega n}^{+/ 0}$ and $\epsilon_{\omega n}^{0 /-}$ defined as

$$
e_{n}^{0}\left(\epsilon_{\omega n}^{+/ 0}\right)=e_{n}^{-}\left(\epsilon_{\omega n}^{0 /-}\right)=\omega,
$$

when $\omega \gg R_{c}^{+/ 0} \equiv n_{0} C_{n}^{+}+p_{0} C_{p}^{0}$ and $4 \exp (U / k T) n_{0} C_{n}^{0}$ $\gg R_{c}^{0 /-} \equiv n_{0} C_{n}^{0}+p_{0} C_{p}^{-}$. On the other hand, $S \cong 0$ for $\omega \ll R_{c}^{+/ 0}$ and $\omega \ll R_{c}^{0 /-}$. These relations clearly demonstrate the correspondence mentioned above so far as the correlated states with an appropriately large $U$ are concerned.

The theoretical result may tempt us to directly extend expression (27) deduced for multiple species of monovalent states to the divalent states as well as to test its applicability. The corresponding expression can be written as

$$
\begin{aligned}
S= & C_{n}^{+} D^{+/ 0}\left(\epsilon_{\omega n}^{+/ 0}\right)\left(\frac{1}{1+2 R_{c}^{+/ 0} / \omega}\right)^{1-\alpha^{+/ 0}} \\
& +C_{n}^{0} D^{0 /-}\left(\epsilon_{\omega n}^{0 /-}\right)\left(\frac{1}{1+2 R_{c}^{0 /-} / \omega}\right)^{1-\alpha l^{0 /-}} .
\end{aligned}
$$

We have made numerical simulation studies of illumination evolution to examine the accuracy of Eq. (A2). In the calculation, a Gaussian distribution of the dangling bond states was chosen, and the nonequilibrium occupation statistics for the correlated states were precisely taken into account. As stated above, Eq. (A1) can be safely used for a larger ratio $n_{0} / p_{0}$. The concentration ratio at a given illumination level is determined by charge neutrality condition. An implication of the neutrality condition is that a large ratio may be the case in $a$-Si:H materials containing a large density of valence band tail states below the dark Fermi level, although measurements of electron-controlled MPC that we are concerned about cannot be applied to determine their distribution and 


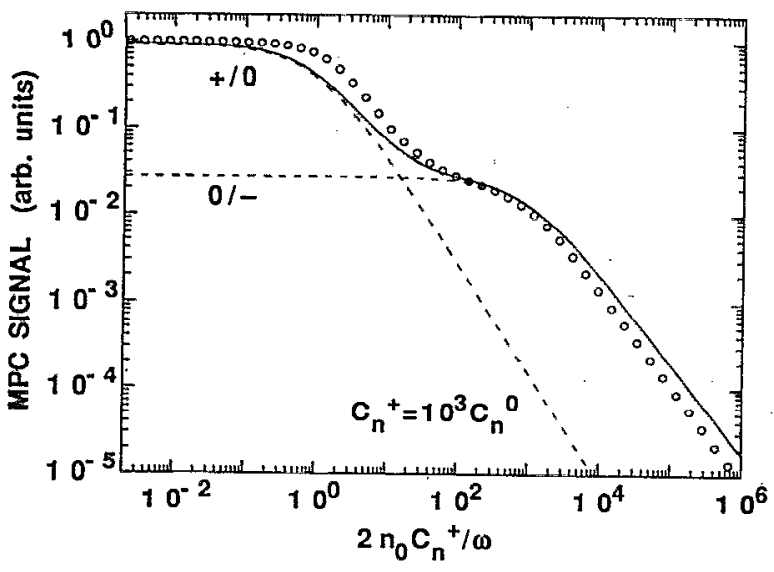

FIG. 7. MPC signal $S$ for dangling bond states with a correlation energy $U=0.4 \mathrm{eV}$ as a function of the ratio $2 n_{\mathrm{v}} C_{n}^{+} / \omega$. The density of states consists of a Gaussian distribution $D^{+/ 0}(\epsilon) \propto \exp \left[-\left(\epsilon-\epsilon^{+/ 0}\right) 2 s^{2}\right\}$. In the calculation, $\epsilon^{+/ 0}=\epsilon_{c}-0.9 \mathrm{eV}, s=0.15 \mathrm{eV}, \epsilon_{g}=1.8 \mathrm{eV}, \epsilon_{F}=\epsilon^{+/ 0}+U / 2$ $=\epsilon_{\mathrm{c}}-0.7 \mathrm{eV}, n_{0} / p_{0}=10^{4}, N_{c, v}-10^{20} \mathrm{~cm}^{-3}, C_{n, p}^{0}=10^{-8} \mathrm{~cm}^{3} \mathrm{~s}^{-1}$, $C_{n}^{+}=C_{p}^{-}=10^{-5} \mathrm{~cm}^{3} \mathrm{~s}^{-1}, \omega=1 \mathrm{~s}^{-1}$, and $\epsilon_{\mathrm{w} n}^{+/ 0}=\epsilon_{\mathrm{c}}-0.5 \mathrm{eV}$ are chosen. Solid curve is the plot calculated from Eq. (A2).

capture coefficients. For the sake of simplicity in the present simulation, the evolution curve associated with the dangling bond states was calculated under a constant concentration ratio (taken as $n_{0} / p_{0} \geqslant 10$ ) instead of incorporating the tail states. Practically, a variation of $n_{0} / p_{0}$ with the illumination level can affect the MPC behavior and also the experimental determination of capture coefficients. This point will be discussed later. An example of calculated results is presented in Fig. 7. It is readily found from the figure that the deviation from the plot calculated from Eq. (A2) is only within a factor of $2 .{ }^{28}$ We have also confirmed that, even when varying the dangling bond parameters, the concentration ratio and the frequency, as well as the temperature, Eq. (A2) is applicable and is valid.

Finally, we shall address what conditions justify an experimental determination of capture coefficients from the electron-controlled MPC. The estimation of $C_{n}^{+}$and that of $C_{n}^{0}$ is possible when $n_{0} / p_{0} \gg C_{p}^{0} / C_{n}^{+}$and when $n_{0} / p_{0} \gg C_{p}^{-} / C_{n}^{0}$, respectively. 'The first condition is consistent with a general trend that a charged center is more efficient in capturing a carrier than a neutral one due to Coulomb attraction. However, it might be marginal that the second condition is completely true. Assuming that a hole capture by $D^{-}$is dominated by a diffusive process, as discussed in Sec. IV A, leads to a relation that $C_{p}^{-} / C_{n}^{+}=\mu_{p} / \mu_{n}$. In this situation, it can be said from the results of measurements described in Sec. III B that our experimental approach for deriving $C_{n}^{0}$ is verified when $n_{0} / p_{0} \gg 10$.

\section{APPENDIX B: MODULATED PHOTOCURRENT IN A TIME-OF-FLIGHT CONFIGURATION}

Here, we will consider charge carrier transport in a TOF measurement configuration, for instance, $p-i-n$ diodes. Photocarriers are generated locally by a strongly absorbed light at one face of the sample and separated by the electric field that consists of an internally built-in and an externally applied field. Consequently, only one type of carrier drifts across the sample and the measured current reflects trapping and release kinetics of these carriers.

The rate equations for electron transport are expressed for the uniform field $E$ by

$$
\begin{gathered}
\frac{\partial n}{\partial t}+\mu_{n} E \frac{\partial n}{\partial x}+\sum_{j} \int_{\epsilon_{v}}^{\epsilon_{c}} d \epsilon D^{j}(\epsilon)\left\{n C_{n}^{j}\left[1-f^{j}(\epsilon)\right]\right. \\
\left.-e_{n}^{j}(\epsilon) f^{j}(\epsilon)\right\}=g \delta(x), \\
\frac{d f^{j}(\epsilon)}{d t}=n C_{n}^{j}\left[1-f^{j}(\epsilon)\right]-e_{n}^{j}(\epsilon) f^{j}(\epsilon),
\end{gathered}
$$

where $x$ is the direction between the contacts $(x=0$ and $x=d)$. For a sinusoidally varying illumination $g=g_{0}+g_{\omega} \cos (\omega t)$, solution forms are written by $n=n_{0}+\operatorname{Re}\left[n_{\omega} \exp (i \omega t)\right]$ for the electron concentration and, similarly, $J=J_{0}+\operatorname{Re}\left[J_{\omega} \operatorname{cxp}(i \omega t)\right]$ for the resulting photocurrent. Solving Eqs. (B1) and (B2) yields the modulated part of concentration

$$
n_{\omega}=\frac{g_{\omega}}{\mu_{n} E} \exp \left(-\frac{(i \omega+N) x}{\mu_{n} E}\right)
$$

where the rate $N$ corresponds to that given by Eq. (9) with $f_{0}^{j}=n_{0} C_{n}^{j} /\left(n_{0} C_{n}^{j}+e_{n}^{j}\right)$. The current continuity permits formulating the modulated photocurrent as ${ }^{19}$

$$
J_{\omega}=\frac{e \mu_{n} E}{d} \int_{0}^{d} d x n_{\omega}=e g_{\omega} \frac{1-\exp \left[-(i \omega+N) t_{\text {transit }}\right]}{\cdot(i \omega+N) t_{\text {transit }}},
$$

where $t_{\text {transit }}=d / \mu_{n} E$ denotes the free electron transit time.

Two classes of TOF measurements can be distinguished according to the relative magnitude of the transit time $t_{\text {transit }}$ compared to the trapping time $t_{\text {trap }}$ defined as

$$
\frac{1}{t_{\text {trap }}} \equiv \operatorname{Re}(N)=\sum_{j} \int_{\epsilon_{t n}^{j}}^{\epsilon_{\omega n}^{j}} d \epsilon C_{n}^{j} D^{j}(\epsilon)
$$

In arriving at the above formulation, we used an approximation that $\operatorname{Re}\left[i \omega /\left(i \omega+e_{n}^{j}\right)\right]=\Theta\left(\epsilon_{\omega n}^{j}-\epsilon\right)$, where $\Theta(\epsilon)$ denotes a unit step function. ${ }^{5,7,9}$ When $t_{\text {transit }}$ is much longer than $t_{\text {trap }}$, Eq. (B4) can be reduced to

$$
J_{\omega}=\frac{e g_{\omega}}{t_{\text {transit }}} \frac{1}{i \omega+N},
$$

indicating that the analysis similar to the modulated photoconductivity $\sigma_{\omega}$ described in Sec. II can be successfully applied. $^{29} \mathrm{~A}$ physical implication in the condition that $t_{\text {transit }} \gg t_{\text {trap }}$ is that photoelectrons can experience a great number of trapping and release events during the transit. On the other hand, the expression becomes

$$
J_{\omega}=e_{\omega}\left(1-\frac{(i \omega+N) t_{\text {transit }}}{2}\right),
$$

when the opposite relation that $t_{\text {transit }} \ll t_{\text {trap }}$ stands so that photoelectrons preferentially exit the sample rather than be trapped in the defect states. 
${ }^{1}$ R. J. Schwartz, J. L. Gray, G. B. Turner, D. Kanani, and H. Ullal, in Proceedings of the 17th IEEE Photovoltaic Specialists Conference (IEEE, New York, 1984), p. 369.

${ }^{2}$ M. J. Powell, C. van Berkel, A. R. Franklin, S. C. Deane, and W. I. Milne, Phys. Rev. B 45, 4160 (1992).

${ }^{3}$ R. A. Street, Hydrogenated Amorphous Silicon (Cambridge University Press, Cambridge, 1991), pp. 104-130.

${ }^{4}$ J. D. Cohen, J. Non-Cryst. Solids 114, 381 (1989).

${ }^{5}$ H. Oheda, J. Appl. Phys. 52, 6693 (1981).

${ }^{6}$ G. Schumm and G. H. Bauer, Phys. Rev. B 39, 5311 (1989).

${ }^{7}$ R. Brüggemann, C. Main, J. Berkin, and S. Reynolds, Philos. Mag. B 62, 29 (1990).

${ }^{8}$ J. P. Kleider, C. Longeaud, and O. Glodt, J. Non-Cryst. Solids 137\&138, 447 (1991).

${ }^{9}$ C. Longeaud and J. P. Kleider, Phys. Rev. B 45, 11672 (1992).

${ }^{10}$ J. P. Kleider, C. Lungeaud, and P. Roca i Cabarrocas, J. Appl. Phys. 72, 4727 (1992).

${ }^{11}$ F. Zhong and J. D. Cohen, Phys. Rev. Lett. 71, 597 (1993).

${ }^{12}$ C. Longeaud and J. P. Kleider, Phys. Rev. B 48, 8715 (1993).

${ }^{13}$ K. Winer, Phys. Rev. B 41, 12150 (1990).

${ }^{14} \mathrm{~K}$. Pierz, W. Fuhs, and H. Mell, Philos. Mag. B 63, 123 (1991).

${ }^{\text {is }}$ G. Schumm and G. H. Bauer, Philos. Mag. B 64, 515 (1991).

${ }^{16}$ M. J. Powell and S. C. Deane, Phys. Rev. B 48, 10815 (1993).

${ }^{17}$ J. G. Simmons and G. W. Taylor, Phys. Rev. B 4, 502 (1971).

${ }^{18}$ J. Stuke, J. Non-Cryst. Solids 97\&98, 1 (1987).

${ }^{19}$ T. Tiedje, in Semiconductor and Semimetals, edited by J. I. Pankove (Academic, New York, 1984), Vol. 21, Pt. C, pp. 721-765.
${ }^{20}$ J. J. Thomson, Philos. Mag. 47, 337 (1924).

${ }^{21}$ D. Adler, M. Silver, A. Madan, and W. Czubatyj, J. Appl. Phys. 51, 6429 (1980).

${ }^{22}$ M. P. Langevin, Ann. Chem. Phys. 28, 433 (1903).

${ }^{23} \mathrm{~K}$. Hattori, H. Okamoto, and X. Hamakawa, J. Non-Cryst. Solids 114, 687 (1989).

${ }^{24}$ N. F. Mot, Philos. Mag. B 63, 3 (1991).

${ }^{25} \mathrm{H}$. Okamoto, K. Hattori, and Y. Hamakawa, J. Non-Cryst. Solids 137\&138, 627 (1991).

${ }^{26}$ J. -K. Lee and E. A. Schiff, Phys. Rev. Lett. 68, 2972 (1992).

${ }^{27}$ In Ref. 16, the energy $k T^{*}$ is estimated to be about $100 \mathrm{meV}$ from the defect density data given in Ref. 14 and by assuming that the charged defects largely outnumber the neutral defects even at low doping levels. This assumption may not be valid as can be seen by Fig. 6 of Ref. 16 .

${ }^{28}$ It can be seen in Fig. 7 that replacing the parameter $2 R_{c}^{+10}$ by $R_{c}^{+10}$ in Eq. (A2) gives a better fit to the simulation result. This adjustment is specific to $+/ 0$ transitions in divalent dangling bond states. However, it is plausible to estimate the capture coefficients by applying Eq. (A2) as well as Eq. (27), without correction, so far as an order of their magnitude is concerned.

${ }^{29}$ Discussion in Appendix B neglects diffusion and surface- or interfacerecombination effects in TOF experiments. If the measurements were carried out at extremely low fields, then their contributions would also become significant and affect defect distribution reconstructed by means of Eq. (26). 